\title{
Caractérisation et étude structurale de $\mathrm{NaHo}\left(\mathrm{PO}_{3}\right)_{4}$
}

\author{
J. Amami ${ }^{1}$, K. Horchani ${ }^{1}$, D. Merle ${ }^{2}$ et M. Férid ${ }^{1}$ \\ 1 Laboratoire des Procédés Chimiques, Institut National de Recherche Scientifique et \\ Technique, BP. 95, 2050 Hammam-Lif, Tunisie \\ ${ }^{2}$ Centre de Diffractométrie UCB Lyon 1, Bâtiment Jules Raulin, 43 Bd. 11 novembre 1918, \\ Villeurbanne 69622, France
}

\begin{abstract}
Crystals of $\mathrm{NaHo}\left(\mathrm{PO}_{3}\right)_{4}$ have been grown by the flux technique and characterized by X-ray diffraction and infrared spectroscopy. Single crystal structure of $\mathrm{NaHo}\left(\mathrm{PO}_{3}\right)_{4}$ has been solved, it crystallizes in the monoclinic $\mathrm{P} 2_{1} / \mathrm{n}$ space group with lattice parameters : $a=9,699(4), b=13,0441(5), c$ $=7,1614(3) \AA, \beta=89,314(1)^{\circ}, \mathrm{Z}=4$. The crystal structure has been refined yielding a final $\mathrm{R}\left(\mathrm{F}^{2}\right)=$ 0.033 for 2064 independent reflections $\left(\mathrm{F}_{\mathrm{o}}^{2} \geq 2 \sigma\left(\mathrm{F}_{\mathrm{o}}^{2}\right)\right)$. The $\mathrm{NaHo}\left(\mathrm{PO}_{3}\right)_{4}$ structure is characterized by helical ribbons $\left(\mathrm{PO}_{3}\right)_{\infty}$ running along the c-axis. The $\mathrm{HoO}_{8}$ and $\mathrm{NaO}_{7}$ polyhedra form zigzag chains with common faces and surrounded by four $\left(\mathrm{PO}_{3}\right)_{\infty}$ chains
\end{abstract}

Résumé. Le polyphosphate $\mathrm{NaHo}\left(\mathrm{PO}_{3}\right)_{4}$ est synthétisé, sous forme de monocristaux, par la méthode de flux; ce composé est caractérisé par diffraction RX et spectroscopie de vibration IR. L'étude structurale de $\mathrm{NaHo}\left(\mathrm{PO}_{3}\right)_{4}$ a montré que ce polyphosphate cristallise dans le système monoclinique, de groupe d'espace $\mathrm{P} 2{ }_{1} / \mathrm{n}$, les paramètres de maille sont : $a=9,699(4), b=13,0441(5), c=7,1614(3) \AA, \beta=$ $89,314(1)^{\circ}, \mathrm{Z}=4$. L'affinement conduit à un facteur de confiance $\mathrm{R}_{1}=3,3 \%$ pour 2064 réflexions indépendantes. La structure de $\mathrm{NaHo}\left(\mathrm{PO}_{3}\right)_{4}$ est caractérisée par une chaîne hélicoïdale $\left(\mathrm{PO}_{3}\right)_{\infty}$ se développant le long de l'axe c. Les polyèdres $\mathrm{HoO}_{8}$ et $\mathrm{NaO}_{7}$ forment des chaînes en zigzag possédant des faces en commun et entourés par quatre chaînes $\left(\mathrm{PO}_{3}\right)_{\infty}$.

\section{INTRODUCTION}

L'étude systématique des phosphates condensés d'éléments monovalents et de lanthanides [1-2] permet de mettre en évidence des composés dont l'intérêt croissant réside dans leurs applications optiques [3-5]. Un intérêt important est porté sur l'holmium pour lequel les processus d'upconversion, d'avalanche et des propriétés laser ont été observés [6].

Le présent travail porte sur la caractérisation et la détermination structurale du polyphosphate d'holmium $\mathrm{NaHo}\left(\mathrm{PO}_{3}\right)_{4}$.

\section{PARTIE EXPÉRIMENTALE}

Le polyphosphate $\mathrm{NaHo}\left(\mathrm{PO}_{3}\right)_{4}$ est synthétisé, sous forme monocristalline à partir d'un mélange de $\mathrm{Na}_{2} \mathrm{CO}_{3}, \mathrm{Ho}_{2} \mathrm{O}_{3}$ et $\mathrm{H}_{3} \mathrm{PO}_{4}$ comme flux. Ce phosphate est caractérisé par diffraction RX sur poudre et 
spectroscopie de vibration IR. Le spectre d'absorption infrarouge a été enregistré à l'aide d'un spectromètre FTIR 2000 (Perkin-Elmer), sur un échantillon pastillé dans du KBr à l'aide d'une presse. La collecte des intensités a été effectuée à l'aide d'un diffractomètre automatique à quatre cercles de type Nonius Kappa CCD utilisant la radiation $\mathrm{K}_{\alpha}$ du molybdène $(\lambda=0,71073 \AA$ ). La structure a été résolue, par exploitation de la fonction tridimensionnelle de Patterson au moyen du programme WinGX [7] utilisant SHELXS 97 [8] puis affinée par SHELXL 97 [9].

\section{RÉSULTATS ET DISCUSSION}

L'étude structurale de $\mathrm{NaHo}\left(\mathrm{PO}_{3}\right)_{4}$ a montré que ce polyphosphate cristallise dans le système monoclinique, de groupe d'espace $\mathrm{P} 2{ }_{1} / \mathrm{n}$; les paramètres de maille sont : $a=9,699(4), b=$ $13,0441(5), c=7,1614(3) \AA, \beta=89,314(1)^{\circ}, Z=4$. L'affinement conduit à un facteur de confiance $\mathrm{R}_{1}=3,3 \%$ pour 1746 réflexions indépendantes. Ce composé est isotype au phosphate condensé de sodium néodyme $\mathrm{NaNd}\left(\mathrm{PO}_{3}\right)_{4}$ [10]. Les conditions de la collecte ainsi que les principaux résultats de l'affinement sont consignés dans le tableau 1. Le tableau 2 donne les principales distances interatomiques dans la structure de $\mathrm{NaHo}\left(\mathrm{PO}_{3}\right)_{4}$.

Tableau 1. Données cristallographiques, conditions de la collecte et résultats de l'affinement de la structure de $\mathrm{NaHo}\left(\mathrm{PO}_{3}\right)_{4}$.

\section{DONNÉES CRISTALLOGRAPHIQUES}

\begin{tabular}{|c|c|}
\hline Formule chimique : & $\mathrm{NaHo}\left(\mathrm{PO}_{3}\right)_{4}$ \\
\hline Système cristallin : monoclinique & $\mathrm{a}=9,699(4), b=13,0441(5), c=7,1614(3) \AA$ \\
\hline \multirow{2}{*}{ Groupe d'espace $: \mathrm{P} 2_{1} / \mathrm{n}$} & $B=89,314(1)^{\circ}$ \\
\hline & $V=950,13 \AA^{3}, Z=4$ \\
\hline $\mathrm{F}(000)=$ & 936 \\
\hline$\rho_{\text {cal }}\left(\mathrm{g} \cdot \mathrm{cm}^{-3}\right)$ & 3,694 \\
\hline Masse molaire $\left(\mathrm{g} \cdot \mathrm{mol}^{-1}\right)$ & 503,8 \\
\hline Coefficient d'absorption $\left(\mathrm{mm}^{-1}\right)$ & 9,55 \\
\hline Dimensions du cristal $\left(\mathrm{mm}^{3}\right)$ & $0,16 \times 0,15 \times 0,2$ \\
\hline Domaine angulaire $\left(^{\circ}\right)$ : & $2,62<\theta<27,45$ \\
\hline Domaine de mesure : & $-12<\mathrm{h}<12,-16<\mathrm{k}<15,-9<1<9$ \\
\hline Nombre de réflexions indépendantes: & 2064 \\
\hline Correction d'absorption : & Intégration \\
\hline Réflexions uniques incluses : & 1746 pour $\mathrm{I}>2 \sigma(\mathrm{I})$ \\
\hline Schéma de pondération WGHT = & $1 /\left[\sigma^{2}\left(\mathrm{~F}_{\mathrm{o}}^{2}\right)+(0,0424 \mathrm{P})^{2}+4,0768 \mathrm{P}\right]$ où $\mathrm{P}=\left(\mathrm{F}_{\mathrm{o}}^{2}+2 \mathrm{~F}_{\mathrm{c}}^{2}\right) / 3$ \\
\hline Nombre de paramètres affinés & 164 \\
\hline Facteurs de reliabilité & $\mathrm{R}=0,033, \mathrm{Rw}=0,078$ \\
\hline
\end{tabular}


Tableau 2. Principales distances inter atomiques dans la structure de $\mathrm{NaHo}\left(\mathrm{PO}_{3}\right)_{4}$.

\begin{tabular}{|c|c|c|c|c|c|c|c|}
\hline \multicolumn{4}{|c|}{ Tétraèdres $\mathrm{PO}_{4}$} & & & & \\
\hline \multicolumn{4}{|c|}{ Tétraèdre autour de $\mathrm{P}_{1}$} & \multicolumn{4}{|c|}{ Tétraèdre autour de $\mathrm{P}_{2}$} \\
\hline $\mathrm{P}_{1^{-}} \mathrm{O}_{4}$ & $1,488(4)$ & $\mathrm{O}_{4}-\mathrm{P}_{1^{-}}-\mathrm{O}_{12}$ & $116,0(3)$ & $\mathrm{P}_{2}-\mathrm{O}_{8}$ & $1,481(4)$ & $\mathrm{O}_{8}-\mathrm{P}_{2}-\mathrm{O}_{7}$ & $117,4(3)$ \\
\hline $\mathrm{P}_{1}-\mathrm{O}_{12}$ & $1,493(5)$ & $\mathrm{O}_{4}-\mathrm{P}_{1}-\mathrm{O}_{3}$ & $108,2(2)$ & $\mathrm{P}_{2}-\mathrm{O}_{7}$ & $1,486(5)$ & $\mathrm{O}_{8}-\mathrm{P}_{2^{-}} \mathrm{O}_{6}$ & $106,1(3)$ \\
\hline $\mathrm{P}_{1}-\mathrm{O}_{9}$ & $1,589(4)$ & $\mathrm{O}_{12}-\mathrm{P}_{1}-\mathrm{O}_{9}$ & $112,3(2)$ & $\mathrm{P}_{2}-\mathrm{O}_{6}$ & $1,571(4)$ & $\mathrm{O}_{7}-\mathrm{P}_{2}-\mathrm{O}_{6}$ & $112,4(3)$ \\
\hline \multirow{3}{*}{$\mathrm{P}_{1}-\mathrm{O}_{6}$} & $1,592(4)$ & $\mathrm{O}_{4}-\mathrm{P}_{1}-\mathrm{O}_{6}$ & $108,4(3)$ & $\mathrm{P}_{2}-\mathrm{O}_{3}$ & $1,600(4)$ & $\mathrm{O}_{7}-\mathrm{P}_{2}-\mathrm{O}_{3}$ & $106,9(2)$ \\
\hline & & $\mathrm{O}_{12}-\mathrm{P}_{1^{-}}-\mathrm{O}_{6}$ & $112,0(3)$ & & & $\mathrm{O}_{8}-\mathrm{P}_{2^{-}} \mathrm{O}_{3}$ & $110,2(2)$ \\
\hline & & $\mathrm{O}_{9}-\mathrm{P}_{1}-\mathrm{O}_{6}$ & $98,3(2)$ & & & $\mathrm{O}_{6}-\mathrm{P}_{2}-\mathrm{O}_{3}$ & $102,9(2)$ \\
\hline \multicolumn{8}{|c|}{ Tétraèdre autour de $\mathrm{P}_{3}$} \\
\hline $\mathrm{P}_{3}-\mathrm{O}_{10}$ & $1,479(5)$ & $\mathrm{O}_{10}-\mathrm{P}_{3}-\mathrm{O}_{2}$ & $119,3(3)$ & \multicolumn{4}{|c|}{ Tétraèdre autour de $\mathrm{P}_{4}$} \\
\hline $\mathrm{P}_{3}-\mathrm{O}_{2}$ & $1,484(4)$ & $\mathrm{O}_{10}-\mathrm{P}_{3}-\mathrm{O}_{1}$ & $111,1(2)$ & $\mathrm{P}_{4}-\mathrm{O}_{5}$ & $1,474(5)$ & $\mathrm{O}_{5}-\mathrm{P}_{4^{-}} \mathrm{O}_{11}$ & $118,0(3)$ \\
\hline $\mathrm{P}_{3}-\mathrm{O}_{1}$ & $1,589(4)$ & $\mathrm{O}_{2}-\mathrm{P}_{3}-\mathrm{O}_{1}$ & $107,9(2)$ & $\mathrm{P}_{4}-\mathrm{O}_{11}$ & $1,484(4)$ & $\mathrm{O}_{5}-\mathrm{P}_{4}-\mathrm{O}_{3}$ & $111,8(2)$ \\
\hline $\mathrm{P}_{3}-\mathrm{O}_{9}$ & $1,591(4)$ & $\mathrm{O}_{10}-\mathrm{P}_{3}-\mathrm{O}_{9}$ & $110,0(2)$ & $\mathrm{P}_{4}-\mathrm{O}_{3}$ & $1,588(4)$ & $\mathrm{O}_{11}-\mathrm{P}_{4}-\mathrm{O}_{3}$ & $107,7(3)$ \\
\hline & & $\mathrm{O}_{2}-\mathrm{P}_{3}-\mathrm{O}_{9}$ & $104,5(2)$ & $\mathrm{P}_{4}-\mathrm{O}_{1}$ & $1,600(4)$ & $\mathrm{O}_{5}-\mathrm{P}_{4^{-}} \mathrm{O}_{1}$ & $105,9(2)$ \\
\hline & & $\mathrm{O}_{1}-\mathrm{P}_{3}-\mathrm{O}_{9}$ & $102,6(2)$ & & & $\mathrm{O}_{11}-\mathrm{P}_{4^{-}} \mathrm{O}_{1}$ & $110,5(2)$ \\
\hline \multicolumn{4}{|c|}{ Polyèdre $\mathrm{HoO}_{8}$} & & & $\mathrm{O}_{3}-\mathrm{P}_{4^{-}} \mathrm{O}_{1}$ & $101,7(2)$ \\
\hline Ho - $\mathrm{O}_{8}$ & $2,302(4)$ & $\mathrm{Ho}-\mathrm{O}_{7}$ & $2,371(4)$ & & & & \\
\hline Ho - $\mathrm{O}_{11}$ & 2,337 (4) & Ho - $\mathrm{O}_{2}$ & $2,391(4)$ & & & & \\
\hline Ho - $\mathrm{O}_{10}$ & $2,354(4)$ & Ho - $\mathrm{O}_{12}$ & $2,458(4)$ & & & & \\
\hline $\mathrm{Ho}-\mathrm{O}_{4}$ & $2,371(4)$ & Ho - $\mathrm{O}_{5}$ & $2,456(4)$ & & & & \\
\hline
\end{tabular}

Dans les tétraèdres $\mathrm{PO}_{4}$ les distances P-O varient de 1,474(5) à 1,600(4) ̊̊ quant aux angles OPO leurs valeurs s'étendent sur l'intervalle 98,3(2)-119,3(3) . Les polyèdres $\mathrm{HoO}_{8}$ sont distordus, isolés les uns des autres, les distances Ho-O vont de 2,302(4) à 2,456(4) $\AA$; La plus courte distance Ho-Ho est de 5,752(4) ̊. Dans une sphère de rayon $3 \AA$, le nombre de coordination de Na est 8 ; les distances $\mathrm{Na}-\mathrm{O}$ vont de 2,365(5) à 3,001(5) $\AA$. (tableau 2). La structure de $\mathrm{NaHo}\left(\mathrm{PO}_{3}\right)_{4}$ est caractérisée par une chaîne hélicoïdale $\left(\mathrm{PO}_{3}\right)_{\infty}$ se développant le long de l'axe c.(fig.1). Les polyèdres $\mathrm{HoO}_{8}$ (fig.2) et $\mathrm{NaO}_{8}$ forment des chaînes en zigzag possédant des faces en commun et entourés par quatre chaînes $\left(\mathrm{PO}_{3}\right)_{\infty}$.

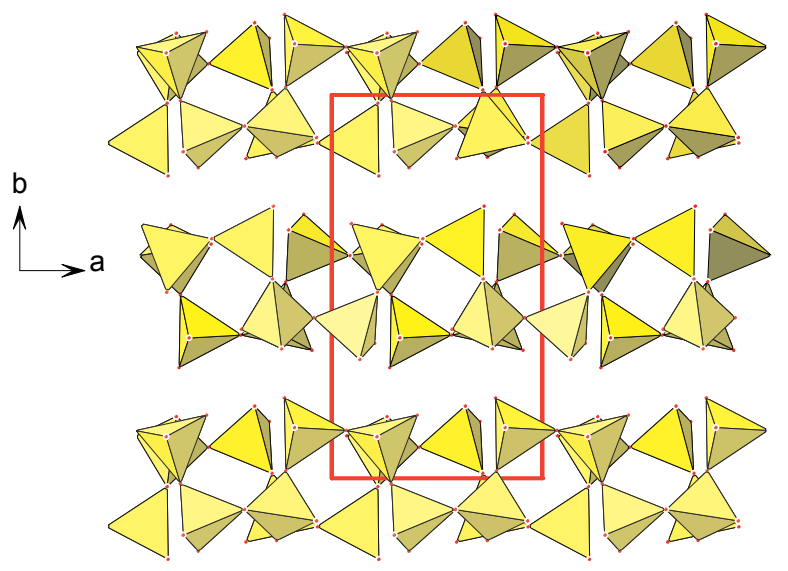

Figure 1. Projection, dans le plan (ab), de la structure (tétraèdres $\left.\mathrm{PO}_{4}\right)$ de $\mathrm{NaHo}\left(\mathrm{PO}_{3}\right)_{4}$. 

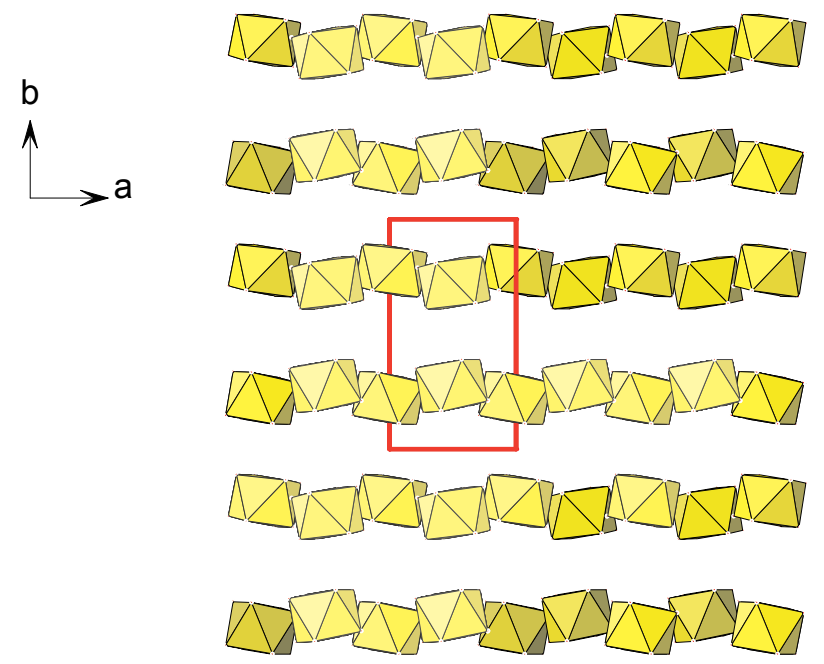

Figure 2. Projection, dans le plan (ab), des polyèdres $\mathrm{HoO}_{8}$ dans la structure de $\mathrm{NaHo}\left(\mathrm{PO}_{3}\right)_{4}$.

Le spectre d'absorption IR de $\mathrm{NaHo}\left(\mathrm{PO}_{3}\right)_{4}$ présente les bandes caractéristiques des vibrations des groupements $\mathrm{O}-\mathrm{P}-\mathrm{O}$ et $\mathrm{PO}_{2}$ des polyphosphates.

Le spectre IR ( fig. 3) montre des bandes de vibrations dans les régions $1352-1215 \mathrm{~cm}^{-1}$ et 1213 $1021 \mathrm{~cm}^{-1}$ correspondant à $v_{\text {as }}\left(\mathrm{PO}_{2}\right)$ et $v_{\mathrm{s}}\left(\mathrm{PO}_{2}\right)$, respectivement, une large bande d'absorption entre 970 et $840 \mathrm{~cm}^{-1}$ correspondant à $v_{\mathrm{as}}(\mathrm{POP})$, le multiplet $\nu_{\mathrm{s}}(\mathrm{POP})$ entre $660-815 \mathrm{~cm}^{-1}$ et le dédoublement des vibrations de déformation des tétraèdres $\mathrm{PO}_{4}$ entre 420 et $620 \mathrm{~cm}^{-1}$.

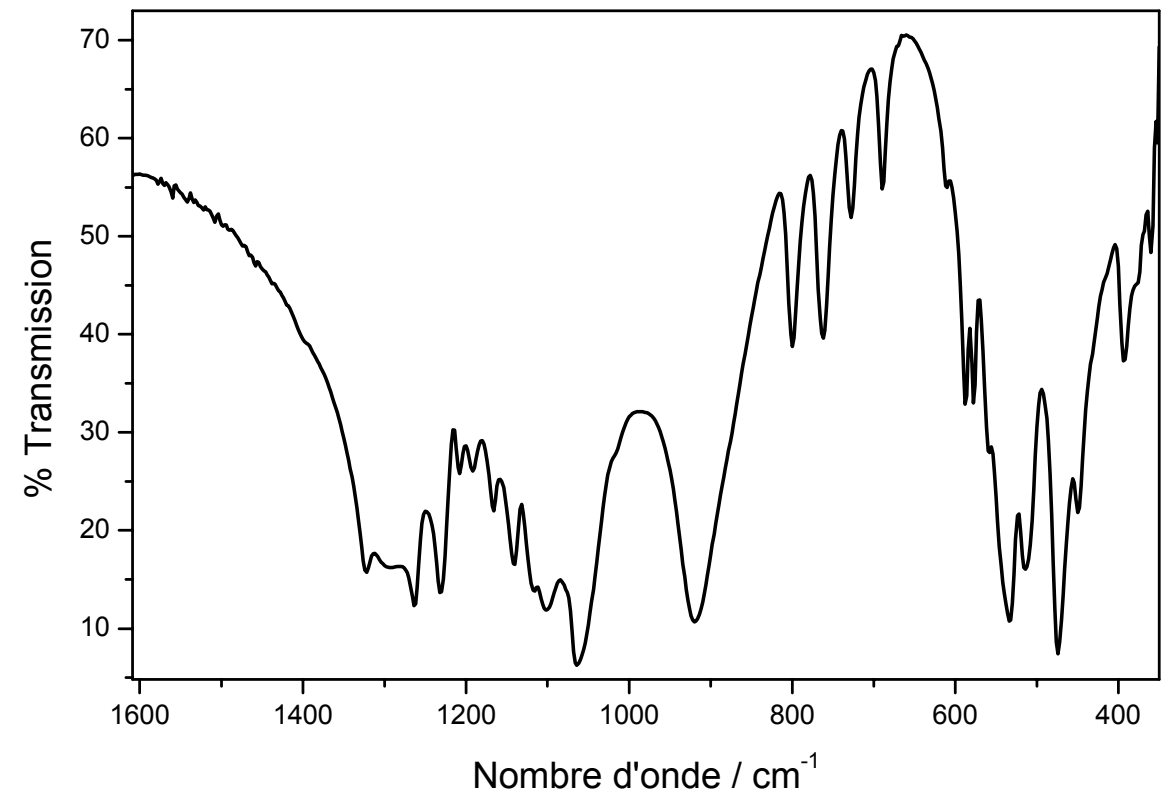

Figure 3. Spectre d'absorption infrarouge de $\mathrm{NaHo}\left(\mathrm{PO}_{3}\right)_{4}$. 


\section{Références}

[1] M. Férid, M. Trabelsi, N.K. Ariguib, J. Solid State Chem. 38 (1981)130

[2] M. Férid, M. Dogguy, M. Trabelsi, N.K. Ariguib, J. Solid State Chem. 53 (1984)149

[3] G. Blasse, B. C. Grabmaier, Luminescent materials, Springer-Verlag (1994)

[4] M. J. Weber, Handbook of Laser Wavelengths, CRC Press (1999)

[5] W. Wolinski, M. Malinowski, R. Wolski, Proceedings of the $9^{\text {th }}$ International Congress, Laser 89 Optoelektronik (1990) 173

[6] G. K. Liu, Y. H. Chen and J. V. Beitz, J. Luminescence. 81 (1999) 7

[7] WinGX - version 1.63, L. J. Farrugia, J.Appl. Cryst. 32 (1999) 837

[8] SHELXS 97, G.M. Sheldrick, Program for Crystal Structure Determination, University of Göettingen, Germany, (1997)

[9] SHELXL 97, G.M. Sheldrick, Program for Crystal Structure Determination, University of Göettingen, Germany, (1997)

[10] H. Koizumi, Acta Cryst. $B 32$ (1976) 2254 